\title{
UM SISTEMA MULTIAGENTE PARA CONTROLE DE UM ASSISTENTE PESSOAL APLICADO A UM AMBIENTE VIRTUAL DE APRENDIZAGEM
}

\author{
Eliseo Reategui - FACED/UFRGS - eliseoreategui@ gmail.com \\ Alexandre Ribeiro - DEIN/UCS - aribeiro@ucs.br \\ Elisa Boff - DEIN/UCS - eboff@ucs.br
}

Resumo. A crescente inserção dos ambientes virtuais no processo de aprendizagem vem trazendo novos desafios a área de Informática na Educação. Entre eles, está o projeto de ambientes que facilitam ao aluno o acesso ao grande volume de informações. Neste contexto, este artigo apresenta um assistente pessoal cujo objetivo é dar apoio aos alunos na utilização dos ambientes virtuais de aprendizagem, fornecendo o suporte apropriado a cada perfil de aluno. $O$ assistente pessoal, modelado em uma abordagem multiagente, faz parte de uma sociedade na qual cada agente controla uma funcionalidade específica, e um agente mediador define qual deles deve entrar em ação a cada momento.

Palavras-chave: sistemas multiagente, agentes de interface, sistemas de recomendação, ambientes virtuais de aprendizagem.

\section{A MULTIAGENT SYSTEM TO CONTROL A VIRTUAL CHARACTER APPLIED TO A VIRTUAL LEARNING ENVIRONMENT}

\begin{abstract}
The use of virtual environments brings new challenges to the area of Computers in Education. Among them, we can highlight the design of system that make it easier for the student to access and manage the large volume of information available. In this context, this paper presents a personal assistant whose goal is to give students support in the use of virtual learning environments, providing the necessary assistance for each student profile. The personal assistant, modeled in a multiagent approach, is part of a society in which each agent controls a specific function, and a mediator agent defines which of them should act in each moment.
\end{abstract}

Keywords: multiagent systems, interface agents, recommender systems, virtual learning environments.

\section{Introdução}

No contexto nacional e internacional, estamos presenciando um verdadeiro "boom" na utilização dos ambientes virtuais de aprendizagem como repositórios de conteúdo e ferramentas de comunicação entre professores e alunos (PALOFF e PRATT, 2001). Alguns alunos mais familiarizados com as tecnologias, rapidamente se apropriam destes 
ambientes computacionais e passam a utilizá-los com grande desenvoltura. Outros menos familiarizados com as tecnologias, podem se mostrar relutantes na utilização destes ambientes (BARAJAS e OWEN, 2000).

Nos países em desenvolvimento nos deparamos ainda com outros problemas, como o fato de que muitos estudantes não têm as habilidades técnicas necessárias para utilizarem os ambientes virtuais de aprendizagem. A escassez de pontos com acesso à internet ou a largura de banda inadequada são outras dificuldades identificadas (SELLAMI, 2005).

Este artigo apresenta um agente de interface personificado cujo objetivo é dar apoio aos alunos na utilização dos ambientes virtuais de aprendizagem, fornecendo o suporte apropriado a cada perfil de aluno. Neste projeto, o agente de interface foi modelado como um sistema multiagente no qual cada agente controla uma funcionalidade específica, e um agente mediador define qual deles deve entrar em ação a cada momento.

O uso de agentes de interface na educação tem se mostrado bastante eficaz nos processos de aprendizagem (PANDZIC et. al. 1999), sendo este um fator motivador para o desenvolvimento do projeto. A partir de informações que se pode obter sobre o aluno e sua utilização dos ambientes, é possível personalizar a comunicação do agente de interface com o aluno, fornecendo a este um ambiente mais voltado aos seus interesses e necessidades (SISON e MASAMICHI, 1998).

Este artigo está organizado da seguinte forma: a próxima seção aborda os sistemas multiagente e propõe a utilização de uma arquitetura específica para o agente de interface. A seção 3 apresenta a arquitetura do sistema multiagente que compõe o assistente pessoal, descrevendo cada um dos agentes projetados. A seção 4 apresenta detalhes de implementação do assistente pessoal desenvolvido para o ambiente virtual de aprendizagem, e a última seção do artigo apresenta considerações finais sobre o projeto.

\section{Os sistemas multiagente (SMA)}

Na concepção de (WOOLDRIDGE, 2002) um agente é uma entidade com capacidade de resolução de problemas encapsulada e possui as propriedades de (i) reatividade, capacidade de manter a interação com o ambiente, como, por exemplo, observar e realizar ações no mundo; (ii) pró-atividade, capacidade de tomar iniciativa, caracterizando um comportamento orientado a objetivos; (iii) habilidade social, ser capaz de realizar ações sociais como comunicação e cooperação, para completarem a resolução de seus problemas, seja ele o auxílio de outros agentes (humanos ou computacionais) ou a decisão de interações apropriadas e (iv) autonomia, possibilidade de operar sem a intervenção direta de outros (possivelmente humanos) agentes e controlar totalmente suas ações e estado interno. Na literatura pode-se encontrar um conjunto maior de características desejáveis a um agente, a saber: Mobilidade, Comunicação, Conhecimento interno, Conhecimento externo, Objetivos e planos, Criatividade, Personalidade, Continuidade temporal, Benevolência, Motivação, Aprendizagem, Cooperação e Veracidade.

Em um SMA os agentes são construídos com seus objetivos e suas capacidades (como, por exemplo, interagir e cooperar) e, a partir daí, são colocados em uma sociedade para 
juntos resolverem um problema. O comportamento de cada sistema emerge do trabalho conjunto desta sociedade. Assim, a área de pesquisa em sistemas multiagente pode ser definida como o estudo de sociedades de agentes autônomos artificiais.

A abordagem multiagente vem sendo utilizada como uma alternativa eficiente na modelagem de ambientes virtuais de aprendizagem mais interativos, dotados de recursos que possibilitem ao aluno um aprendizado mais dinâmico. Além disso, segundo (OLIVEIRA, 1996), os princípios dos SMA mostram-se bastante adequados ao desenvolvimento de ambientes computacionais de ensino, tendo em vista que o problema de ensino-aprendizagem é de natureza social e cooperativa.

\section{Arquitetura do sistema multiagente que compõe o Assistente Pessoal}

O sistema multiagente que compõe o Assistente Pessoal foi organizado em diferentes módulos, cada um destinado a uma função específica, a saber: comunicação, feedback, recomendação, monitoramento e interação. A figura 1 mostra os diferentes agentes que constituem cada um dos módulos.
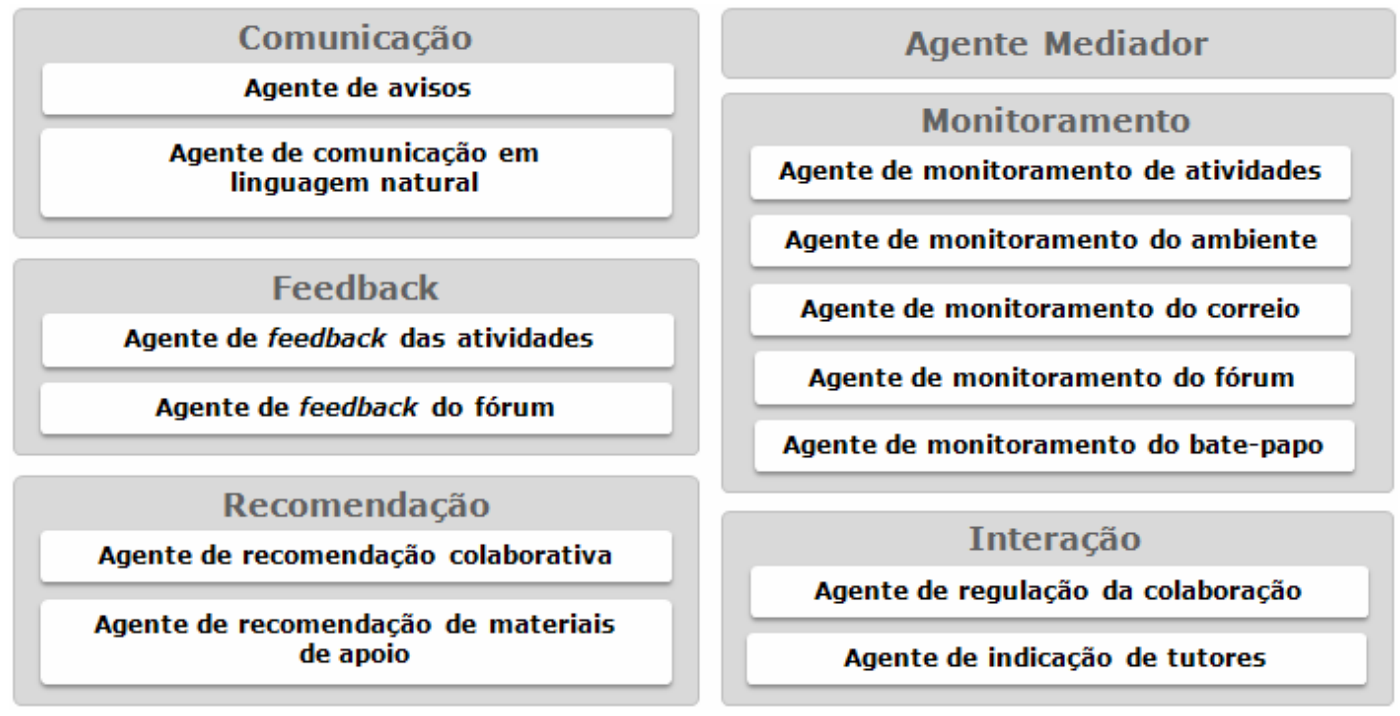

Figura 1: Componentes do sistema multiagente

Alguns destes agentes são extremamente simples, necessitando apenas de um conjunto de consultas a determinadas tabelas de um banco de dados para aportarem seus resultados. São exemplos destes componentes os agentes de feedback e de monitoramento. Já o agente de comunicação em linguagem natural ou o agente de recomendação colaborativa são bastante complexos, necessitando de mecanismos bem mais elaborados para seu funcionamento. As subseções a seguir detalham cada um dos módulos definidos e seus respectivos agentes.

\subsection{O Módulo de Comunicação}

O módulo de comunicação é responsável pela apresentação de avisos importantes ao aluno bem como pela capacidade do personagem em dialogar com o aluno em linguagem natural. Este módulo é composto por dois agentes, apresentados a seguir.

\subsubsection{O agente de avisos}


O agente de avisos tem o objetivo de apresentar recados importantes ao aluno. Dentro do contexto do ambiente virtual de aprendizagem no qual o agente está inserido, duas fontes são consultadas para obtenção dos avisos: o mural dos cursos nos quais o aluno está inscrito, bem como as mensagens instantâneas recebidas pelo aluno e que são internas ao ambiente virtual de aprendizagem.

\subsubsection{O agente de comunicação em linguagem natural}

$\mathrm{O}$ agente de comunicação em linguagem natural utiliza a linguagem de representação AIML - Artificial Intelligence Markup Language, ou Linguagem de Marcação de Inteligência Artificial (WALLACE 2003) - para receber perguntas do aluno e retornar respostas apropriadas. O sistema armazena estímulos (frases e fragmentos) que podem ser utilizados para questionar o assistente, empregando-os na busca por respostas previamente estabelecidas. Vários destes sistemas estão disponíveis na web sob licença GPL. A idéia é que um deles seja empregado e adaptado para as necessidades do portal.

Alguns dos principais marcadores da linguagem são:

$<$ aiml>: tag de início de documento

$<$ categoria >: tag que marca as unidades de conhecimento em uma base

<pattern>: utilizado para conter um padrão que será buscado nos diálogos que os usuários entrarão para se comunicar com o assistente

<template>: contém as respostas às entradas feitas pelo usuário.

Além dos marcadores já existentes para descrever conhecimento em AIML, o agente emprega um mecanismo complementar que permite o controle a mudança de imagens que refletem os diferentes estados de espírito do personagem (feliz, receptivo, incomodado, etc.). Deste modo, ao introduzir uma pergunta ao assistente (i.e. um estímulo), o assistente busca em sua base uma resposta apropriada e a apresenta, trocando de estado quando necessário. Um banco de frases genérico foi desenvolvido para o personagem, falando sobre o ambientes virtuais de aprendizagem e sua utilização. A estrutura deste banco permite que, com bastante facilidade, sejam agregados novos conhecimentos (frases, respostas) relacionados aos diversos assuntos que se queira tratar. A figura 2 mostra um exemplo de interação com o personagem virtual no qual ele responde uma questão específica sobre envio de mensagens pelo correio do ambiente virtual de aprendizagem.

\subsubsection{Agente de notícias}

O agente de notícias tem o objetivo de encaminhar notícias ao usuário. Um mecanismo hierárquico organiza os feeds de notícias e os distribui aos destinatários. O agente é responsável por receber os feeds e "negociar" sua apresentação com outros agentes que também buscam levar os mais diversos tipos de informação ao usuário.

\subsection{O Módulo de Feedback}

Este módulo tem o objetivo de informar o aluno de que o professor, ou outros alunos, deram alguma resposta com relação a alguma atividade realizada. O módulo é composto por dois agentes apresentados a seguir.

\subsubsection{O agente de feedback dos formadores: 3}


Este agente é responsável por avisar o aluno de que o formador revisou/comentou alguma tarefa postada. $\mathrm{O}$ agente consulta a lista de tarefas colocadas no webfólio e verifica se o professor postou algum comentário ainda não consultado pelo aluno, avisando-o apropriadamente.

\subsubsection{O agente de feedback dos alunos: 4}

Este agente tem o mesmo funcionamento do agente de feedback dos formadores (item 3.2.1). No entanto, para determinado aluno, ele verifica a existência de comentários e feedbacks de outros alunos para as atividades postadas.

\subsection{O Módulo de Monitoramento}

O módulo de monitoramento visa supervisionar os bancos de dados do sistema verificando se o aluno está realizando as tarefas propostas e participando de outras atividades, como discussões em fóruns, bate-papo, entre outras. O módulo é composto pelos agentes apresentados nas próximas subseções.

\subsection{2. $O$ agente de monitoramento de atividades}

Este agente é responsável por avisar o aluno que atividades estão pendentes, caso isso ocorra. $\mathrm{O}$ agente consulta a lista de atividades existente no webfólio e verifica se para alguma delas o prazo para postagem está próximo e o aluno ainda não a entregou.

Também devem ser levados em consideração materiais disponibilizados para leitura (ou acesso) e que ainda não tenham sido consultados.

\subsubsection{O agente de monitoramento do correio}

$\mathrm{O}$ agente de monitoramento do correio informa o aluno de mensagens recebidas e ainda não consultadas.

\subsubsection{O agente de monitoramento do bate-papo}

$\mathrm{O}$ agente de bate-papo avisa o aluno de datas de bate-papo próximas da data atual.

\subsubsection{O agente de monitoramento do fórum}

Este agente é responsável por informar o aluno de novas respostas a mensagens suas postadas no fórum, ou a seqüência de discussões das quais o aluno já tenha participado.

\subsubsection{O agente de monitoramento do ambiente}

Quando o aluno não acessa o ambiente regularmente, de acordo com uma taxa de frequiência estabelecida pelo professor no módulo de administração, o agente adverte o aluno que sua participação está muito baixa, e que este deveria participar mais.

\subsection{O Módulo de Recomendação}

O módulo de recomendação tem o objetivo de recomendar materiais de apoio para o aluno como tarefas de reforço à aprendizagem, e também sugerir leituras e outras atividades de acordo com o que é observado na comunidade de alunos. As próximas subseções descrevem com mais detalhe os dois agentes de recomendação.

\subsubsection{O agente de recomendação colaborativa}

Este módulo emprega um sistema de recomendação para identificar e sugerir tópicos de interesse dos alunos. Em tempo real, ele identifica padrões nas leituras e atividades 
complementares mais acessadas pela comunidade de alunos, e utiliza estes padrões para sugerir materiais relacionados aos tópicos de estudo atuais do usuário.

Uma das técnicas mais antigas utilizadas pelos sistemas de recomendação é conhecida como filtragem colaborativa (HERLOCKER, 2004). Esta técnica prevê as preferências de um usuário com base nas preferências de outros com comportamento semelhante. Hoje em dia os sistemas de recomendação já migraram dos laboratórios de pesquisa para o mercado da internet, e podem ser encontrados em grandes websites como o da livraria Amazon.com ou da loja de discos CDNow.com (SCHAFER, 2000) . Na Educação, contudo, não há tantos registros de utilização dos sistemas de recomendação. Geyer-Schulz et al.(2001), por exemplo, exploram o potencial dos sistemas de recomendação na educação com relação à melhoria da comunicação entre professor/aluno, à redução da sobrecarga de informação, à personalização e construção de equipes. Reategui et al. (2007) apresentam um agente pedagógico capaz de fazer recomendação de conteúdos a partir de padrões de navegação identificados nos logs de um ambiente virtual de aprendizagem.

Nosso agente de recomendação utiliza a técnica tradicional de Filtragem Colaborativa, que pode ser descrita em três fases:

1. Cálculo da similaridade de cada usuário do sistema com o usuário alvo (métrica de similaridade).

2. Seleção de um subconjunto de usuários com maiores similaridades (vizinhos).

3. Cômputo das predições considerando os itens acessados/avaliados pelos vizinhos mais próximos.

Esta técnica também é chamada de "k-nearest-neighbor" ou "k-vizinhos mais próximos". No primeiro passo, a definição da similaridade pode ser realizada através de diversos coeficientes, sendo mais comumente aplicado o coeficiente de correlação de Pearson (SCHARDANAN e MAES, 1995).

\subsubsection{O agente de recomendação de materiais de apoio}

Este agente é responsável por recomendar materiais de apoio relacionados a alguma atividade que o aluno não consegue realizar. Isso é feito através de orientações de aula que o professor pode registrar e suas conexões com o material de apoio.

\subsection{O Módulo de Interação}

O módulo de interação é responsável por promover a interação entre alunos em duas situações distintas, para as quais foram concebidos os agentes específicos abaixo descritos.

\subsubsection{O agente de regulação da colaboração}

Slavin (1991) mostrou em vários experimentos as vantagens da colaboração com relação à realização de atividades individualmente. Tais estudos deram origem a outros apontando situações específicas nas quais a colaboração pode ser profícua. Dillenburg (2000), por exemplo, aponta para a necessidade de organização da colaboração entre alunos para que as interações sejam são realmente eficazes:

- Estruturação da colaboração: o professor não apenas cria uma atividade e estabelece os grupos, mas também cria um cenário com várias fases nas quais os 
alunos devem cumprir determinados papéis.

- Regulação da colaboração: um membro externo monitora a interação do grupo e garante que todos os membros participam ativamente.

O agente desenvolvido neste projeto dá suporte à segunda tarefa, ou seja, ele assegura que todos os membros de um grupo colaboram na realização das atividades propostas. A principal dificuldade no monitoramento de atividades de grupo é que muitas destas são desenvolvidas offline, e nestes casos não é possível estabelecer quem foram os membros que efetivamente participaram no seu desenvolvimento. No entanto, quando estas são desenvolvidas em ferramentas especialmente concebidas para atividades colaborativas (e.g. wikis), o monitoramento e alerta a alunos com pouca participação torna-se possível.

\subsubsection{O agente de indicação de tutores}

Em certas situações é importante promover a colaboração dos alunos em atividades individuais, sobretudo para auxiliar alunos com dificuldades. Neste contexto, três requisitos são necessários (BOFF et al., 2007):

- Encontrar um estudante capacitado e disposto a colaborar como tutor;

- Incentivar a criação de um grupo entre o "aluno tutor" e o "aluno em dificuldades";

- Prover ferramentas para permitir a comunicação.

O agente de indicação de tutores busca identificar situações nas quais um aluno poderia se beneficiar da interação com outro aluno tutor. Tal situação poderia ser identificada, por exemplo, quando a data limite para postar uma atividade se aproxima e determinado aluno já consultou a tarefa diversas vezes sem ainda tê-la concluído.

O algoritmo de indicação de tutores utilizado neste trabalho é baseado nos atributos humor, performance, aceitação e número de interações envolvendo o aluno. Tais cálculos envolvem a quantificação de fatores psicológicos e de personalidade em conjunto com o histórico pedagógico do aluno. Em Reategui et al. (2006) mais detalhes sobre este algoritmo podem ser encontrados.

\subsection{O Agente Mediador}

$\mathrm{O}$ agente mediador tem o principal objetivo de organizar as intervenções dos diversos agentes que compõem o assistente pessoal de modo que a comunicação com o usuário não fique desordenada, com intervenções erráticas e descontinuadas. Trata-se portanto de um mecanismo de coordenação centralizado, no qual um único agente tem como função decidir qual dos outros deve entrar em ação.

Através de um mecanismo de pesos, o agente mediador estabelece prioridades entre os agentes, definindo a ordem com que estes podem atuar. Por default, o agente com maior nível de prioridade no sistema é o agente de comunicação em linguagem natural, seguido dos agentes do módulo de monitoramento. No entanto, qualquer formador pode alterar esta ordem de prioridades no contexto de sua(s) disciplina(s), influenciando o modo com que o agente mediador filtra e encaminha aos estudantes as informações consideradas mais importantes. 


\section{Aspectos de Implementação}

Um agente de interface com as características descritas acima encontra-se em desenvolvimento para ser integrado ao portal acadêmico da Universidade de Caxias do Sul, o UCSvirtual. O ambiente virtual de aprendizagem disponível neste portal possui uma estrutura comum a outros AVAs, como por exemplo:

- Mural: presente na página de acesso ao AVA, possibilita ao professor postar recados para a turma;

- Cronograma: página onde é apresentado o planejamento das atividades ao longo da disciplina, contendo uma breve descrição do que será tratado em cada aula, com links para as orientações da aula;

- Projeto da disciplina: dados da disciplina, envolvendo ementa, programa, metodologia, avaliação e bibliografia (básica e complementar);

- Orientações: diretrizes estabelecidas para cada aula sobre a metodologia e avaliação empregadas na turma;

- Participantes: professor(es), alunos e monitor (quando existir). Através da página de participantes, também pode-se enviar mensagens instantâneas aos demais membros da turma;

- Acervo: material disponibilizado pelo professor, podendo ficar no âmbito da turma ou da disciplina, ficando neste último caso acessível aos alunos do conjunto de turmas de uma mesma disciplina;

- Fórum: sistema de fóruns para fomentar discussões e trocas de idéias entre professor e alunos de uma turma;

- Sala de bate-papo: chat para conversas síncronas entre os membros da turma;

- Webfólio: sistema de entrega de trabalhos que permite ao professor dar retorno para o aluno através do próprio ambiente.

$\mathrm{O}$ assistente pessoal foi implementado em AJAX, ficando ativo em quaisquer dos módulos acima listados. No exemplo ilustrado na figura 2, o assistente faz uma saudação ao aluno e lhe apresenta um recado: "A próxima avaliação de matemática será no dia 30 de abril”. Em seguida, responde a algumas questões elaboradas em linguagem natural. 


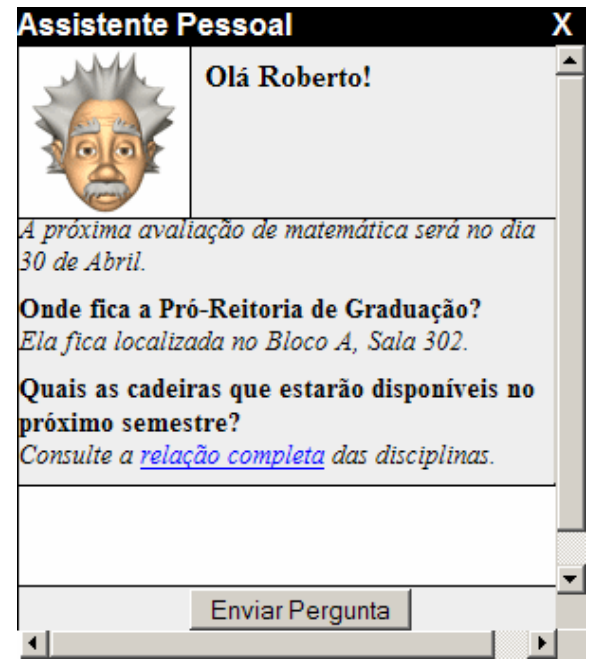

Figura 2: Interação do Assistente Pessoal com aluno fictício de nome Roberto

Note que pelo menos 3 agentes entram em ação para realizar esta interação com o aluno: (1) o agente de avisos traz uma comunicação importante no momento em que o estudante se conecta no sistema; (2) o agente de comunicação em linguagem natural é acionado cada vez que o aluno entra com uma questão e pressiona o botão "Enviar Pergunta"; (3) o agente mediador, operando de forma invisível, seleciona a informação que será levada ao usuário a cada momento advinda dos mais diversos agentes.

A complexidade do sistema aumenta na medida em que mais agentes buscam informações relevantes para encaminhá-las ao agente mediador. Em situações experimentais, não houve degradação da performance do sistema devido à interação constante dos agentes. No entanto, testes de desempenho mais rigorosos deverão ser realizados antes que o sistema seja disponibilizado para acesso por milhares de estudantes.

\section{Considerações Finais}

Os agentes de interface têm sido cada vez mais empregados para criar formas de interação amigáveis com o usuário, introduzindo componentes afetivos que alteram a forma com que o usuário percebe sua comunicação com a máquina. Nos anos 1990, Pattie Maes (1994) já apontava que através dos agentes de interface, o usuário poderia interagir com os sistemas computacionais e ser engajado em um processo cooperativo no qual homem e máquina poderiam iniciar a comunicação um com o outro, monitorar eventos e realizar tarefas. Tal forma de interação era fundamentada na metáfora de um ajudante que colaborava com o usuário no mesmo ambiente de trabalho.

Neste trabalho, foi proposto um agente de interface capaz de atuar como assistente do aluno em um ambiente virtual de aprendizagem. Além de consistir em um fator motivacional para os alunos, o que pode ter um papel fundamental nos processos de aprendizagem (HUITT, 2001), o agente de interface proposto oferece serviços bastante úteis, respondendo questões, monitorando a realização das atividades e alertando o aluno de eventuais atrasos, recomendando materiais de apoio e promovendo a interação entre alunos. 
Em Pan et al (2005) os autores apresentam um ambiente virtual de aprendizagem mediado por personagens virtuais no qual estes têm um papel fundamentalmente motivacional. A principal diferença destes com o modelo de personagem apresentado neste trabalho é que, além de servir com um elemento motivacional, nosso agente de interface é baseado em serviços (alertas, monitoramento, recomendações, etc), buscando dar apoio às atividades do usuário.

A utilização de um agente pedagógico junto aos ambientes virtuais de aprendizagem reforça a importância da dimensão sócio-afetiva nos componentes da interface do sistema. Na educação, a ênfase dada a esta dimensão torna-se ainda mais importante, sabendo-se da relevância da afetividade e das trocas sociais nos processos de aprendizagem (VYGOTSKY, 1987). Em um manifesto, Picard et al. (2004) argumentam que nos últimos anos muitas evidências foram encontradas apontando que cognição e afetividade juntas guiam o comportamento racional, a busca de fatos na memória, a criatividade e a tomada de decisão. Enfatizam a necessidade de se desenvolver teorias e tecnologias nas quais cognição e afetividade são integradas de modo apropriado.

Apesar de todos os benefícios relacionados ao uso dos agentes de interface, sua utilização pode eventualmente perturbar o usuário habituado a uma interface na qual não existe nenhum mediador. Dentro desta perspectiva, o design destes agentes ainda possui muitos desafios, tais como decidir qual o nível de controle a ser delegado ao agente e como desenvolver a confiança do usuário no agente.

\section{Referências}

BARAHAS, M., OWEN, M. (2000). Implementing Virtual Learning Environments: Looking for Holistic Approach. Educational Technology \& Society 3(3) 2000. Disponível em: http://ifets.fit.fraunhofer.de/periodical/vol_3_2000/barajas.html

BOFF, E., VICARI, R. M., FLORES, C. D., REATEGUI, E. B. (2007) A Probabilistic Agent to support Collaboration in a Medical Learning Environment. In: 13th Portuguese Conference on Artificial Intelligence, Guimarães. 4th Workshop on Multi-Agent Systems: Theory and Applications, 2007.

DILLENBURG, P. 2000. Virtual Learning Environments. Proc. of EUN Conference 2000 - Workshop on Virtual Learning Environments. Disponível em: http://tecfa.unige.ch/tecfa/publicat/dil-papers-2/Dil.7.5.18.pdf

GEYER-SCHULZ, A., HAHSLER, M., JAHN, M. (2001). Educational and scientific recommender systems: Designing the information channels of the virtual university. International Journal of Engineering Education, 17(2), 153-163.

HERLOCKER, J. L. et al. (2004). Evaluating Collaborative Filtering Recommender Systems. ACM Transactions on Information Systems, New York, v.22, n.1, p. 5-53. Jan.

HUITT, W. (2001). Motivation to learn: An overview. Educational Psychology Interactive. Valdosta, GA: Valdosta State University. Disponível em: http://chiron.valdosta.edu/whuitt/col/motivation/motivate.html

MAES, P. (1994). Agents that reduce work and information overload. Communications 
of the ACM, 37(7):31-40.

OLIVEIRA, F. M.; VICARI, R. M. (1996) “Are learning systems distributed or social systems.” In: European Conference on AI in Education. Lisbon: [s.n].

PALOFF, R. M., PRATT, K. (2001). Lessons from the Cyberspace Classroom. 17th Annual Conference on Distance Teaching and Learning. Madison, Wisconsin, August 8-10, 2001.

PAN, Z., ZHU, J., ZHANG, M., HU, W. Collaborative Virtual Learning Environment Using Synthetic Characters. Advances in Web-Based Learning - ICWL 2005. Lecture Notes in Computer Science, Volume 3583. Bernlin/Heidelberg: Springer. 2005.

PANDZIC, I. S., OSTERMANN, J. and MILLEN, D. (1999). User Evaluation: Synthetic Talking Faces for Interactive Services. The Visual Computer, Springer Verlag, vol. 15. pp. 330-340.

PICARD, R. W., PAPERT, S., BENDER, W., BLUMBERG, B., BREAZEAL, C., CAVAllO, D., MACHOVER, T., RESNICK, M., ROY, D., STROHECKER, C. Affective Learning: A manifesto. BT Technology Journal, Vol 22, No 4. October 2004

REATEGUI, Eliseo; BOFF, Elisa; CERON, Rafael Fernando; VICCARI, Rosa Maria. Um Agente Animado Sócio-Afetivo para Ambientes de Aprendizagem. Revista Brasileira de Informática na Educação - Edição Especial Aprendizagem Colaborativa. v. 14 n.3. Setembro-Dezembro de 2006. p27-38.

REATEGUI, E. ; BOFF, Elisa ; CAMPBELL, J. A. . Using a Virtual Character in Personalized Recommendations. In: Technical Symposium on Computer Science Education, 2007, Covington, Kentucky, USA. Proceedings of ACM SIGCSE 2007, 2007. p. 180-184.

SCHAFER, J.B. et al. (2000). Recommender Systems in E-Commerce. In: ACM CONFERENCE ON E-COMMERCE, 2., 2000, Minneapolis. Proceedings... New York: ACM Press, 2000.

SELLAMI, H.M. Some difficulties in distance learning in developing countries. ICALT 2005. Fifth IEEE International Conference on Volume Advanced Learning Technologies, 5-8 July 2005. Page(s): 1005 - 1006.

SHARDANAND, U; MAES, P. (1995). Social information filtering: Algorithms for automating "word of mouth", In: Proceedings of Human Factors in Computing Systems, p. $210-217$.

SISON, R. e MASAMICHI, S. (1998). Student Modeling and Machine Learning. International Journal of Artificial Intelligence in Education, 9, 128-158.

SLAVIN, R.E. (1991) Cooperative learning: theory, research and practice. Englewoods Cliffs, NJ: Prentice Hall.

VYGOTSKY, L. S. The collected works of L. S. Vygotsky. New York: Plenum Press, c1987-c1999 v. 1-6.

WALLACE, R. (2003). "The Elements of AIML Style", ALICE A. I. Foundation.

WOOLDRIDGE, M. (2002) An introduction to multiagent systems. Chichester: J. 
Wiley, 2002. 348 p.

V. 6 № 1, Julho, 2008 